\title{
Negative differential conductance observed in electron field emission from band gap modulated amorphous-carbon nanolayers
}

\author{
W. M. Tsang, ${ }^{\text {a) }}$ S. J. Henley, V. Stolojan, and S. R. P. Silva \\ Nanoelectronics Centre, Advanced Technology Institute, University of Surrey, Guildford GU2 7XH, \\ United Kingdom
}

(Received 5 July 2006; accepted 20 September 2006; published online 6 November 2006)

\begin{abstract}
Amorphous-carbon $(a-\mathrm{C})$-based quantum confined structures were synthesized by pulsed laser deposition. In these structures, electrons are confined in a few nanometer thick $s p^{2}$ rich $a$-C layer, which is bound by the vacuum barrier and a $3 \mathrm{~nm}$ thick $s p^{3}$ rich $a-\mathrm{C}$ base layer. In these structures anomalous field emission properties, including negative differential conductance and repeatable switching effects, are observed when compared to control samples. These properties will be discussed in terms of resonant tunneling and are of great interest in the generation and amplification of high-frequency signals for vacuum microelectronics and fast switching devices. (C) 2006 American Institute of Physics. [DOI: 10.1063/1.2378492]
\end{abstract}

The operational speed of solid-state electronic devices is limited by the saturation velocity of electrons $\left(\sim 10^{5} \mathrm{~ms}^{-1}\right)$ due to the lattice scattering, while the electron velocity in vacuum can approach the speed of light, $3 \times 10^{8} \mathrm{~ms}^{-1}$. Hence, vacuum microelectronic devices are attractive for high-speed and high-frequency applications. ${ }^{1}$ Electron field emission (FE) materials can be used to fabricate these devices. ${ }^{2}$ The amplifier and oscillator are two key components for high-speed electronics, both of which require an element, such as a resonant tunnel diode (RTD), to provide negative differential conductance (NDC) for operation. Hence, quantum-well (QW) structures showing NDC in their FE process are extremely promising and have attracted a lot of research interest. ${ }^{3-7}$ Most of these studies were focused on the $\mathrm{GaN}$ or Si-based quantum well systems, perhaps because of the early success of applying these materials in RTDs. However, the fabrication processes of these materials (e.g., low-pressure metal organic chemical vapor deposition for $\mathrm{GaN}$ ) are expensive and require a high process temperature. In this work, the FE properties of an amorphous carbon $(a-\mathrm{C})$-based QW structure using pulsed laser ablation of a graphite target at room temperature are studied. We show the NDC and the switching effects in the FE measurements. This work is inspired by our recent success in implementing a RTD using the $a-\mathrm{C}$ system for high-speed switching devices ${ }^{8}$ and by the outstanding physical properties of carbon related materials, such as high electron mobility, high breakdown field, high thermal conductivity, and low threshold fields for electron emission. ${ }^{9,10}$

Figure 1 shows the energy band structure of the $a$-C nanolayers. The layers were prepared by the ablation of a graphite target $(99.999 \%$ purity) using $248 \mathrm{~nm}$ pulsed UV excimer laser (Lambda Physik LPX 210i) at a chamber pressure of $\sim 10^{-7}$ mbar. The substrates used were $n$-type (100) Si wafers (resistivity $\leqslant 0.05 \Omega \mathrm{cm}$ ), with samples growth without any substrate heating. The band gap $\left(E_{g}\right)$ of the $a$-C layers was modulated by the laser fluence. First, a $3 \mathrm{~nm}$ thick $s p^{3}$ rich $\left(85 \% s p^{3}\right.$ and $\left.E_{g}=2.8 \mathrm{eV}\right) a$-C layer was deposited on the $\mathrm{Si}$ with the laser fluence of $20 \mathrm{~J} / \mathrm{cm}^{2}$, which served as the bottom potential barrier for the structure. This

${ }^{a)}$ Electronic mail: wmtsang @alumni.cuhk.net was followed by another $s p^{2}$ rich $a$-C layer $\left(50 \% s p^{3}\right.$ and $E_{g}=1.5 \mathrm{eV}$ ) with thicknesses of 3, 4, and $6 \mathrm{~nm}$, using a laser fluence of $4 \mathrm{~J} / \mathrm{cm}^{2}$ and served as the "well" layer. The $s p^{2}$ rich surface layer is bound by the $s p^{3}$ rich layer at the back contact, with a barrier height of $0.65 \mathrm{eV}$, and a vacuum barrier of $4.0 \mathrm{eV}$ (electron affinity of carbon) on top of the device. Hence, it forms an asymmetric QW structure. In addition, single layered $6 \mathrm{~nm}$ thick $a$-C layers, either $s p^{2}$ rich or $s p^{3}$ rich, and double layered $a-\mathrm{C}$ structures with opposite layer arrangements $\left(s p^{3}-s p^{2}-\mathrm{Si}\right)$ are also fabricated as control samples. The details of the fabrication process and characterization of these $a$-C layers are published elsewhere. ${ }^{8,11}$ The FE properties of the samples were studied at room temperature in a high vacuum chamber with a base pressure better than $5 \times 10^{-4} \mathrm{~Pa}$. The current-field $(I-F)$ characteristics were measured using a "sphere-to-plane" electrode configuration,
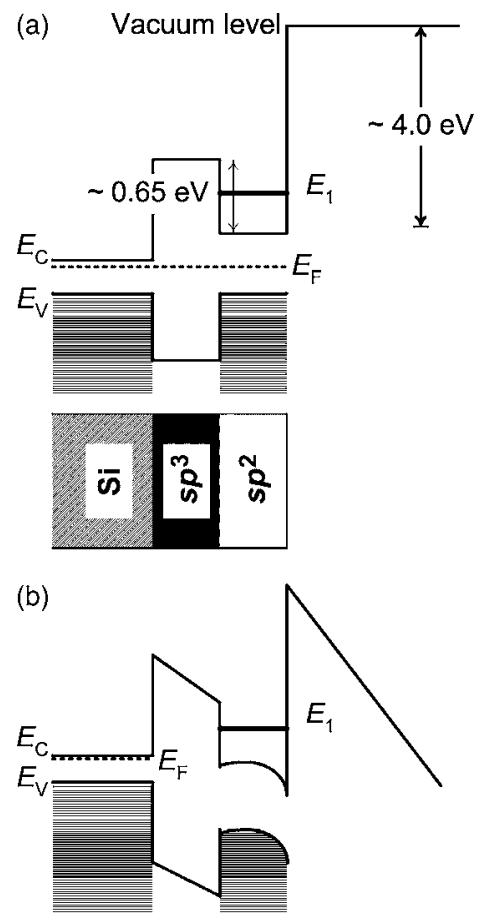

FIG. 1. Electron energy band structure of $s p^{2}-s p^{3}$-Si layer: (a) absence of external field and (b) present of external field. 

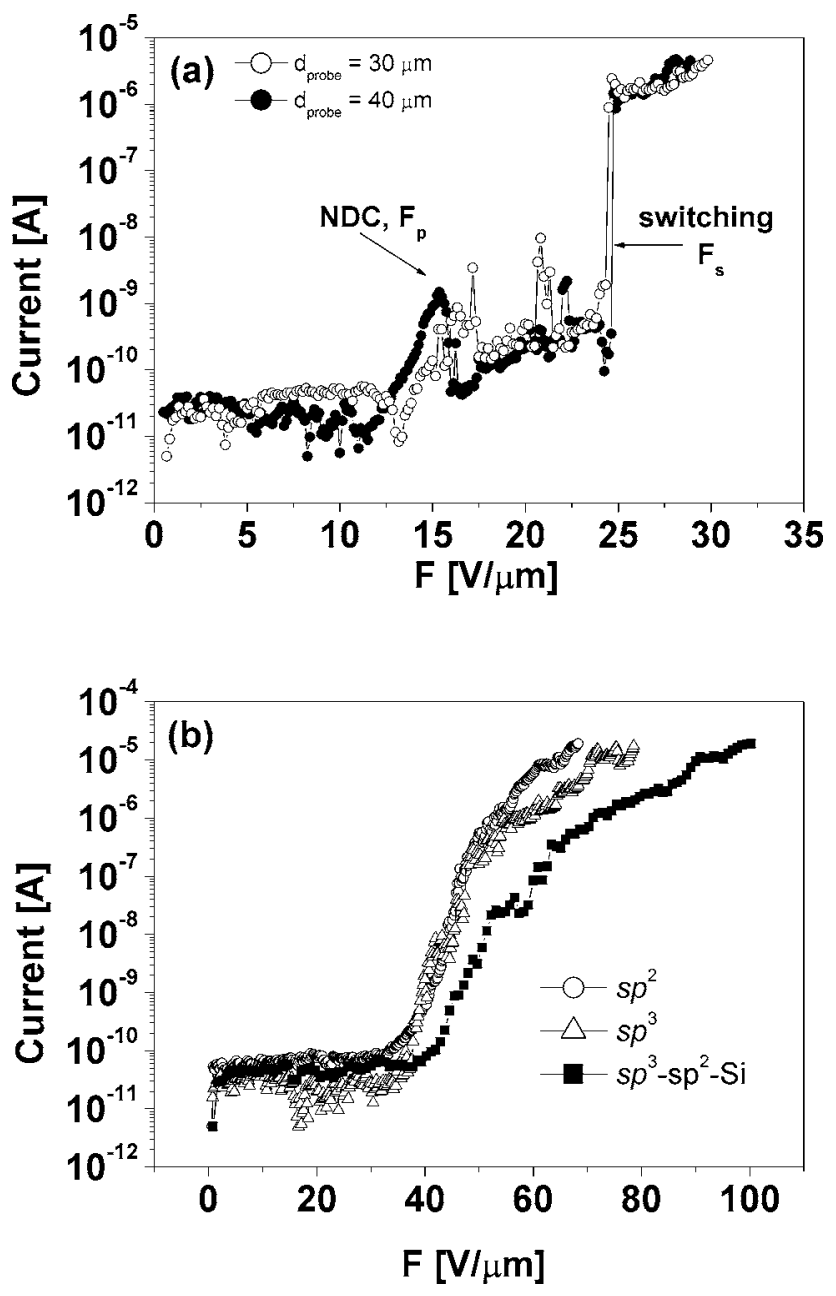

FIG. 2. Electron emission characteristics of the samples with structure of (a) $s p^{2}-s p^{3}-\mathrm{Si}$ (with anode gap distances of 30 and $40 \mu \mathrm{m}$ ) and (b) $s p^{2}, s p^{3}$, and $s p^{3}-s p^{2}-\mathrm{Si}$.

with a $5 \mathrm{~mm}$ diameter stainless-steel ball anode. The measurements were preformed in different areas of each sample and the high voltage was cycled up and down three times to check the homogeneity and repeatability of each large area sample produced. The applied electric field was obtained by dividing the applied voltage by the gap distance.

A typical $I-F$ characteristic of the sample with a quantum well structure of $s p^{2}-s p^{3}-\mathrm{Si}$ is plotted on a logarithmic scale in Fig. 2(a), where the thickness of the $s p^{2}$ and $s p^{3}$ layers is also $3 \mathrm{~nm}$. The characteristics of this sample are extremely different from the conventional Fowler-Nordheimtype-tunneling curve as shown for the control samples in Fig. 2(b). NDC with a peak at the field $\left(F_{p}\right)$ of $15 \mathrm{~V} / \mu \mathrm{m}$ and an abrupt increasing of emission current over four orders of magnitude swing (switching effect) at a field $\left(F_{s}\right)$ of $25 \mathrm{~V} / \mu \mathrm{m}$ is observed with anode gap distances of 30 and $40 \mu \mathrm{m}$, at different regions of the same sample for three successive measurements. The variation of $F_{p}$ and $F_{s}$ with respect to the thickness of the $s p^{2}$ layer is shown in Fig. 3. These results suggest that the characteristics of the $s p^{2}-s p^{3}$ -Si structure do not originate from a conditioning effect in the $a$-C films. We propose that these characteristics can be attributed to resonant tunneling across the $\mathrm{QW}$ structure.

As shown schematically in Fig. 1, the conduction band minima $\left(E_{C}\right)$ of the $s p^{2}$ layer was split by the QW structure and creates a subband $E_{1}$. As the thickness of the $s p^{2}$ rich Downloaded 30 Mar 2009 to 131.227 .178 .132 . Redistribution subje

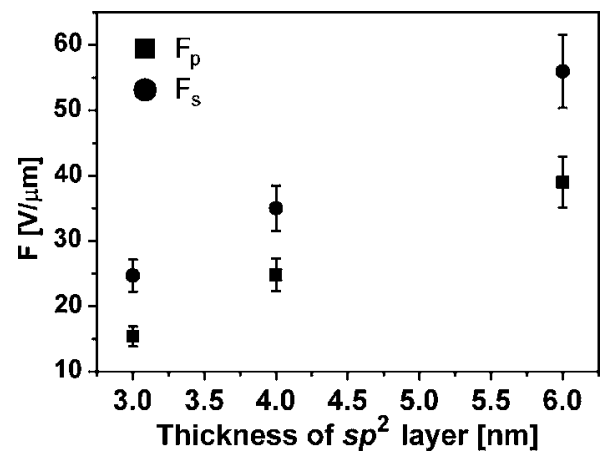

FIG. 3. Variation of $F_{p}$ and $F_{s}$ against the width of $s p^{2}$ layer in the $s p^{2}-$ $s p^{3}-\mathrm{Si}$ structure.

$a$-C layer is only a few nanometers in thickness and the $E_{g}$ is $\sim 1.5 \mathrm{eV}$, the applied electric field can effectively penetrate through the $s p^{2}$ layer and fully deplete this thin semiconductor film. The fully depleted $s p^{2} a$-C layer could create electric fields much greater than the macroscopic field external to it. ${ }^{12-14}$ Similar to the space charge induced band bending model for amorphous materials in which the Si substrate acts as the electron source, ${ }^{12-15}$ Semet et al. ${ }^{5}$ and Wang et al. ${ }^{7}$ proposed an identical hypothesis for GaN based double layered structures. We postulate electron injection from $\mathrm{Si}$ into the $s p^{2}$ "well layer" through the $3 \mathrm{~nm}$ thick $s p^{3}$ layer by field penetration. This then allows for electrons to accumulate in the subenergy levels in $s p^{2}$ layer, which can be emitted under the right conditions. Although, the accumulation of electrons in the $s p^{2}$ well will screen out the external field and reduce the field penetration, this space charge concentration in the $s p^{2}$ layer will give a space potential and create an upward energy band bending in the $s p^{2}$ layer, as shown in Fig. 1(b) at the front positive end of the sample. According to the equation derived by Semet et al. ${ }^{5}$ the space potential energy by the electrons accumulated in the first subenergy level $\left(E_{1}\right)$ can be estimated as $V_{\mathrm{SC}}$ $=\left(\rho_{0} / 4 \varepsilon_{0} \varepsilon_{r}\right)\left\{(w / \pi)^{2} \sin 2(\pi x / w)+w x-x^{2}\right\}$, where $w$ is the thickness of the $s p^{2}$ layer and $\rho_{0}$ is the space charge density (is proportional to the energy difference between the first and the second subenergy level). The $E_{2}-E_{1}$ is calculated to be $0.45 \mathrm{eV}\left(E_{1}=0.2 \mathrm{eV}\right.$ and $\left.E_{2}=0.65 \mathrm{eV}\right)$ for the effective mass $m^{*} \sim 0.06, \varepsilon_{r} \sim 4$, and $w=3 \mathrm{~nm}$ which is further confirmed by modeling. ${ }^{8,15}$ Hence, the average value of the band bending in the $s p^{2}$ layer attributed to the space potential is $\sim 0.2 \mathrm{eV}$ and is comparable to $E_{1}$. When $E_{c}$ of the $\mathrm{Si}$ substrate is aligned with the first confined energy $E_{1}$ by this band bending, resonant tunneling can occur and hence the emission current will increase. However, when the field $\left(>F_{p}\right)$ is further increased, the energy level $E_{1}$ will align with the forbidden gap of the Si substrate. Hence, the condition of resonant tunneling disappears and the emission current decreases with increase of electric field, and hence, NDC is observed.

However, as the field is further increased, at a higher empirically predicted value $F_{s}$, the valence band maxima $\left(E_{V}\right)$ of the Si substrate will be aligned with energy level $E_{1}$. Resonant tunneling can then once more commence and therefore an abrupt increase in the emission current is achieved. Since the electron concentration in the valence band is very high, as it is nearly a full band, the condition for resonant tunneling is still valid with further increase in the electric field. Identical $a$-C structures should be fabricated on to AlP license or copyright; see http://apl.aip.org/apl/copyright.jsp 
$p$-type silicon substrates (or other substrate materials) to verify the above discussions. As shown in Fig. $3, F_{p}$ and $F_{s}$ are monotonically increasing with width $(d)$ of the well $\left(s p^{2}\right.$ layer). Although, the oxygen content, its change upon current oxidation and local thickness fluctuations will all slightly modify the FE properties, from the transmission electron microscopy measurements any thickness fluctuations are significantly less than the well thickness. ${ }^{11}$ Hence, it is expected that $F_{p}$ and $F_{s}$ should monotonically decrease with $d$ as $E_{1} \propto 1 / d^{2}$ for an infinite QW. Such a reduction could be the result of a decrease in field penetration as the thickness of the $s p^{2}$ layer increases and the dependence of the local field enhancement on thickness of the $a$-C layer is a possible explanation. Although the layer-thickness effect on the FE properties of $s p^{2}-s p^{3}-\mathrm{Si}$ structure is under investigation, it shows the possibility of tailoring the position of the resonant tunneling peak $F_{p}$ and switching-on field $F_{s}$, by varying the layer thickness.

In summary, we demonstrate negative differential conductance and switching effects in electron field emission from $a-\mathrm{C}$ based quantum confined structures $\left(s p^{2}-s p^{3}-\mathrm{Si}\right)$. The positions of the NDC and the switching fields are dependent on the thickness of the $s p^{2}$ layer. As the thickness of the $s p^{2}$ layers increasing from 3 to $6 \mathrm{~nm}$, the $F_{p}$ and $F_{s}$ increase from 15 to $39 \mathrm{~V} / \mu \mathrm{m}$ and from 25 to $56 \mathrm{~V} / \mu \mathrm{m}$, respectively. These anomalous FE properties in term of the resonant tunneling effect could be useful for high-speed electronics such as oscillators and fast switching devices, which makes $a$-C very attractive as a large area electronics thin film.
The authors thank the financial support from the EPSRC in the form of a Portfolio Partnership Award. One of the authors (W.M.T.) acknowledges the financial support from the Croucher Foundation.

${ }^{1}$ W. Zhu, Vacuum Microelectronics (Wiley, New York, 2001), Chap. 1, 7.

${ }^{2}$ K. B. K. Teo, E. Minoux, L. Hudanski, F. Peauger, J. P. Schnell, L. Gangloff, P. Legagneux, D. Dieumegard, G. A. J. Amaratunga, and W. I. Milne, Nature (London) 437, 968 (2005).

${ }^{3}$ K. L. Jensen, IEEE Trans. Electron Devices 38, 2337 (1991).

${ }^{4}$ V. Litovchenko, A. Evtukh, Yu. Kryuchenko, N. Goncharuk, O. Yilmazoglu, K. Mutamba, H. L. Hartnagel, and D. Pavlidis, J. Appl. Phys. 96, 867 (2004).

${ }^{5}$ V. Semet, Vu Thien Binh, J. P. Zhang, J. Yang, M. Asif Khan, and R. Tsu, Appl. Phys. Lett. 84, 1937 (2004).

${ }^{6}$ A. Ishida, Y. Inoue, and H. Fujiyasu, Appl. Phys. Lett. 86, 183102 (2005).

${ }^{7}$ R. Z. Wang, X. M. Ding, B. Wang, K. Xue, J. B. Xu, H. Yan, and X. Y. Hou, Phys. Rev. B 72, 125310 (2005).

${ }^{8}$ S. Batttacharyya, S. J. Henley, E. Mendoza, L. Gomez-Rojas, J. Allam, and S. R. P. Silva, Nat. Mater. 5, 19 (2006).

${ }^{9}$ J. Robertson, Mater. Sci. Eng., R. 271, 1 (2002).

${ }^{10}$ S. R. P. Silva, Properties of Amorphous Carbon, EMIS Datareviews Series No. 29 (INSPEC, London, 2003), 135.

${ }^{11}$ V. Stolojan, P. Moreau, S. J. Henley, M. J. Goringe, and S. R. P. Silva, Ultramicroscopy 106, 346 (2006).

${ }^{12}$ R. D. Forrest, A. P. Burden, S. R. P. Silva, L. K. Cheah, and X. Shi, Appl. Phys. Lett. 73, 3784 (1998).

${ }^{13}$ G. A. J. Amaratunga and S. R. P. Silva, Appl. Phys. Lett. 68, 2529 (1996).

${ }^{14}$ S. R. P. Silva, J. D. Carey, G. Y. Chen, D. C. Cox, R. D. Forrest, C. H. P. Poa, R. C. Smith, Y. F. Tang, and J. M. Shannon, IEE Proc.: Circuits Devices Syst. 151, 489 (2004).

${ }^{15}$ V. Stolojan, P. Moreau, M. J. Goringe, and S. R. P. Silva, Appl. Phys. Lett. 88, 122109 (2006). 